\title{
Impact of Displaying Inpatient Pharmaceutical Costs at the Time of Order Entry: Lessons From a Tertiary Care Center
}

\author{
Sarah J. Conway, MD ${ }^{1,2 \star}$, Daniel J. Brotman, MD,2, Brian L. Pinto, PharmD, MBA', David Merola ${ }^{3,4}$, \\ Leonard S. Feldman, MD ${ }^{1,2}$, Redonda G. Miller, MD, MBA ${ }^{1,2}$, Kenneth M. Shermock, PharmD, PhD ${ }^{3,4}$
}

\begin{abstract}
BACKGROUND: A lack of cost-conscious medication use is a major contributor to excessive healthcare expenditures in the inpatient setting. Expensive medicines are often utilized when there are comparable alternatives available at a lower cost. Increasing prescriber awareness of medication cost at the time of ordering may help promote cost-conscious use of medications in the hospital.
\end{abstract}

OBJECTIVE: To evaluate the impact of cost messaging on the ordering of 9 expensive medications.

DESIGN: Retrospective analysis of an institutional cost-transparency initiative.

SETTING: A 1145-bed, tertiary care, academic medical center.

PARTICIPANTS: Prescribers who ordered medications through the computerized provider order entry system at the Johns Hopkins Hospital.

METHODS: Interrupted time series and segmented regression models were used to examine prescriber ordering be- fore and after implementation of cost messaging for 9 highcost medications.

RESULTS: Following the implementation of cost messaging, no significant changes were observed in the number of orders or ordering trends for intravenous (IV) formulations of eculizumab, calcitonin, levetiracetam, linezolid, mycophenolate, ribavirin, and levothyroxine. An immediate and sustained reduction in medication utilization was seen in 2 drugs that underwent a policy change during our study, IV pantoprazole and oral voriconazole. IV pantoprazole became restricted at our facility due to a national shortage (-985 orders per 10,000 patient days; $P<0.001$ ), and oral voriconazole was replaced with an alternative antifungal in oncology order sets ( -110 orders per 10,000 patient days; $P=0.001$ ).

CONCLUSIONS: Prescriber cost transparency alone did not significantly influence medication utilization at our institution. Active strategies to reduce ordering resulted in dramatic reductions in ordering. Journal of Hospital Medicine 2017;12: 639-645. @ 2017 Society of Hospital Medicine
Secondary to rising healthcare costs in the United States, broad efforts are underway to identify and reduce waste in the health system. ${ }^{1,2}$ A recent systematic review exhibited that many physicians inaccurately estimate the cost of medications. ${ }^{3}$ Raising awareness of medication costs among prescribers is one potential way to promote high-value care.

Some evidence suggests that cost transparency may help prescribers understand how medication orders drive costs. In a previous study carried out at the Johns Hopkins Hospital, fee data were displayed to providers for diagnostic laboratory tests. ${ }^{4}$ An $8.6 \%$ decrease (95\% confidence interval [CI], $-8.99 \%$ to $-8.19 \%$ ) in test ordering was observed when costs were displayed vs a $5.6 \%$ increase (95\% CI, $4.90 \%$ to $6.39 \%$ ) in ordering when costs were not displayed during a 6-month intervention period $(P<0.001)$. Conversely, a similar study that investigated the impact of cost transparency on inpatient imaging utilization did not demonstrate a significant

'Department of Medicine, The Johns Hopkins Hospital, Baltimore, Maryland; ${ }^{2}$ The Johns Hopkins School of Medicine, Baltimore, Maryland; ${ }^{3}$ Department of Pharmacy, The Johns Hopkins Hospital, Baltimore, Maryland; ${ }^{4}$ Center for Medication Quality and Outcomes, The Johns Hopkins Hospital, Baltimore, Maryland.

*Address for correspondence and reprint requests: Sarah Johnson Conway, MD, 600 North Wolfe Street / Meyer 8-145, Baltimore, MD 21287; Telephone 410-502-2128; Fax 410-502-0923 E-mail: sjohn207@jhmi.edu

Received: November 9, 2016; Revised: February 18, 2017; Accepted: February 26, 2017

2017 Society of Hospital Medicine DOI 10.12788/jhm.2779 influence of cost display. ${ }^{5}$ This suggests that cost transparency may work in some areas of care but not in others. A systematic review that investigated price-display interventions for imaging, laboratory studies, and medications reported 10 studies that demonstrated a statistically significant decrease in expenditures without an effect on patient safety. ${ }^{6}$

Informing prescribers of institution-specific medication costs within and between drug classes may enable the selection of less expensive, therapeutically equivalent drugs. Prior studies investigating the effect of medication cost display were conducted in a variety of patient care settings, including ambulatory clinics, ${ }^{7}$ urgent care centers, ${ }^{8}$ and operating rooms, ${ }^{9,10}$ with some yielding positive results in terms of ordering and $\operatorname{cost}^{11,12}$ and others having no impact. ${ }^{13,14}$ Currently, there is little evidence specifically addressing the effect of cost display for medications in the inpatient setting.

As part of an institutional initiative to control pharmaceutical expenditures, informational messaging for several high-cost drugs was initiated at our tertiary care hospital in April 2015. The goal of our study was to assess the effect of these medication cost messages on ordering practices. We hypothesized that the display of inpatient pharmaceutical costs at the time of order entry would result in a reduction in ordering.

\section{METHODS}

Setting, Intervention, and Participants

As part of an effort to educate prescribers about the high 
cost of medications, 9 intravenous (IV) medications were selected by the Johns Hopkins Hospital Pharmacy and Therapeutics Committee as targets for drug cost messaging. The intention of the committee was to implement a rapid, lowcost, proof-of-concept, quality-improvement project that was not designed as prospective research. Representatives from the pharmacy and clinicians from relevant clinical areas participated in preimplementation discussions to help identify medications that were subjectively felt to be overused at our institution and potentially modifiable through provider education. The criteria for selecting drug targets included a variety of factors, such as medications infrequently ordered but representing a significant cost per dose (eg, eculizumab and ribavirin), frequently ordered medications with less expensive substitutes (eg, linezolid and voriconazole), and high-cost medications without direct therapeutic alternatives (eg, calcitonin). From April 10, 2015, to October 5, 2015, the computerized Provider Order Entry System (cPOE), Sunrise Clinical Manager (Allscripts Corporation, Chicago, IL), displayed the cost for targeted medications. Seven of the medication alerts also included a reasonable therapeutic alternative and its cost. There were no restrictions placed on ordering; prescribers were able to choose the high-cost medications at their discretion.

Despite the fact that this initiative was not designed as a research project, we felt it was important to formally evaluate the impact of the drug cost messaging effort to inform future quality-improvement interventions. Each medication was compared to its preintervention baseline utilization dating back to January 1, 2013. For the 7 medications with alternatives offered, we also analyzed use of the suggested alternative during these time periods.

\section{Data Sources and Measurement}

Our study utilized data obtained from the pharmacy order verification system and the cPOE database. Data were collected over a period of 143 weeks from January 1, 2013, to October 5, 2015, to allow for a baseline period (January 1, 2013, to April 9, 2015) and an intervention period (April 10, 2015, to October 5, 2015). Data elements extracted included drug characteristics (dosage form, route, cost, strength, name, and quantity), patient characteristics (race, gender, and age), clinical setting (facility location, inpatient or outpatient), and billing information (provider name, doses dispensed from pharmacy, order number, revenue or procedure code, record number, date of service, and unique billing number) for each admission. Using these elements, we generated the following 8 variables to use in our analyses: week, month, period identifier, drug name, dosage form, weekly orders, weekly patient days, and number of weekly orders per 10,000 patient days. Average wholesale price (AWP), referred to as medication cost in this manuscript, was used to report all drug costs in all associated cost calculations. While the actual cost of acquisition and price charged to the patient may vary based on several factors, including manufacturer and payer, we chose to use AWP as a generalizable estimate of the cost of acquisition of the drug for the hospital.

\section{Variables}

"Week" and "month" were defined as the week and month of our study, respectively. The "period identifier" was a binary variable that identified the time period before and after the intervention. "Weekly orders" was defined as the total number of new orders placed per week for each specified drug included in our study. For example, if a patient received 2 discrete, new orders for a medication in a given week, 2 orders would be counted toward the "weekly orders" variable. "Patient days," defined as the total number of patients treated at our facility, was summated for each week of our study to yield "weekly patient days." To derive the "number of weekly orders per 10,000 patient days," we divided weekly orders by weekly patient days and multiplied the resultant figure by 10,000 .

\section{Statistical Analysis}

Segmented regression, a form of interrupted time series analysis, is a quasi-experimental design that was used to determine the immediate and sustained effects of the drug cost messages on the rate of medication ordering. ${ }^{15-17}$ The model enabled the use of comparison groups (alternative medications, as described above) to enhance internal validity.

In time series data, outcomes may not be independent over time. Autocorrelation of the error terms can arise when outcomes are more similar at time points closer together than outcomes at time points further apart. Failure to account for autocorrelation of the error terms may lead to underestimated standard errors. The presence of autocorrelation, assessed by calculating the Durbin-Watson statistic, was significant among our data. To adjust for this, we employed a Prais-Winsten estimation to adjust the error term $\left(\varepsilon_{t}\right)$ calculated in our models.

Two segmented linear regression models were used to estimate trends in ordering before and after the intervention. The presence or absence of a comparator drug determined which model was to be used. When only single medications were under study, as in the case of eculizumab and calcitonin, our regression model was as follows:

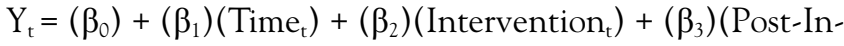
tervention Time $\left._{\mathrm{t}}\right)+\left(\varepsilon_{\mathrm{t}}\right)$

In our single-drug model, $Y_{t}$ denoted the number of orders per 10,000 patient days at week "t"; Time ${ }_{t}$ was a continuous variable that indicated the number of weeks prior to or after the study intervention (April 10, 2015) and ranged from -116 to 27 weeks. Post-Intervention Time t $_{\text {was }}$ a continuous variable that denoted the number of weeks since the start of the intervention and is coded as zero for all time periods prior to the intervention. $\beta_{0}$ was the estimated baseline number of orders per 10,000 patient days at the beginning of the study. $\beta_{1}$ is the trend of orders per 10,000 patient days per week during the preintervention period; $\beta_{2}$ represents an 
TABLE. Summary of the Analyzed High-Cost Medications and Associated Monthly Hospital Expenditures

\begin{tabular}{|c|c|c|}
\hline Medication & Average Spent Monthly & Message \\
\hline Voriconazole injection & $\$ 56,000$ & $\begin{array}{l}\text { Infectious disease approval required. Voriconazole injection cost: } \$ 152 \text { per } 200 \mathrm{mg} \text { dose vs } \$ 47 \text { per } 200 \mathrm{mg} \text { oral dose. Consider } \\
\text { enteral therapy after achieving therapeutic trough on IV. Consult pharmacy for oral dose conversion (not 1:1 in children). }\end{array}$ \\
\hline Levetiracetam injection & $\$ 30,000$ & $\begin{array}{l}\text { Levetiracetam injection cost: } \$ 82 \text { per } 1500 \mathrm{mg} \text { dose vs } \$ 10 \text { per } 1500 \mathrm{mg} \text { oral dose. Consider enteral therapy at 1:1 dose conversion } \\
\text { unless clinically contraindicated. }\end{array}$ \\
\hline Levothyroxine injection & $\$ 26,000$ & $\begin{array}{l}\text { Levothyroxine injection cost: } \$ 55 \text { per } 100 \text { mcg dose vs } \$ 0.80 \text { per } 200 \text { mcg oral dose. Consider enteral therapy at a } 50 \% \text { dose conver- } \\
\text { sion unless clinically contraindicated. }\end{array}$ \\
\hline Linezolid injection & $\$ 26,000$ & $\begin{array}{l}\text { Linezolid injection cost: } \$ 290 \text { per } 600 \text { mg dose vs } \$ 180 \text { per } 600 \mathrm{mg} \text { oral dose. Consider enteral therapy at 1:1 dose conversion unless } \\
\text { clinically contraindicated. }\end{array}$ \\
\hline Eculizumab injection & $\$ 315,000$ & $\begin{array}{l}\text { Not for institutional review board studies. Insurance authorization required prior to dispensing first dose. Eculizumab injection cost: } \\
\$ 22,000 \text { per } 900 \text { mg IV dose. }\end{array}$ \\
\hline Pantoprazole injection & $\$ 24,000$ & $\begin{array}{l}\text { Supply is critically low due to a national shortage. Refer to online formulary for IV restriction criteria. Pantoprazole injection cost: } \$ 6 \text { per } \\
40 \mathrm{mg} \text { dose vs } \$ 4 \text { per } 40 \mathrm{mg} \text { oral dose. Consider enteral therapy at 1:1 dose conversion unless clinically contraindicated. }\end{array}$ \\
\hline Calcitonin injection & $\$ 14,000$ & $\begin{array}{l}\text { Calcitonin injection cost: } \$ 1107 \text { per } 200 \text { units. For hypercalcemia, consider alternatives including hydration, bisphosphonates, and/or } \\
\text { corticosteroids. Contact clinical pharmacy specialist for recommendations. }\end{array}$ \\
\hline Ribavirin inhalation & $\$ 169,000$ & Ribavirin inhalation cost: $\$ 13,023$ per $6 \mathrm{~g}$ dose. \\
\hline Mycophenolate Mofetil injection & $\$ 46,000$ & $\begin{array}{l}\text { Mycophenolate Mofetil injection cost: } \$ 210 \text { per } 1 \mathrm{gm} \text { dose vs } \$ 16 \text { per } 1 \mathrm{gm} \text { oral dose. Consider enteral therapy at 1:1 dose conversion } \\
\text { unless clinically contraindicated. }\end{array}$ \\
\hline
\end{tabular}

estimate of the change in the number of orders per 10,000 patient days immediately after the intervention; $\beta_{3}$ denotes the difference between preintervention and postintervention slopes; and $\varepsilon_{t}$ is the "error term," which represents autocorrelation and random variability of the data.

As mentioned previously, alternative dosage forms of 7 medications included in our study were utilized as comparison groups. In these instances (when multiple drugs were included in our analyses), the following regression model was applied:

$Y_{t}=\left(\beta_{0}\right)+\left(\beta_{1}\right)\left(\right.$ Time $\left._{t}\right)+\left(\beta_{2}\right)\left(\right.$ Intervention $\left._{t}\right)+\left(\beta_{3}\right)($ Post-Intervention Time $)+\left(\beta_{4}\right)($ Cohort $)+\left(\beta_{5}\right)($ Cohort $)\left(\right.$ Time $\left._{t}\right)+$ $\left(\beta_{6}\right)($ Cohort $)($ Intervention $)+\left(\beta_{7}\right)($ Cohort $)($ Post-Intervention Time $\left._{t}\right)+\left(\varepsilon_{t}\right)$

Here, 3 coefficients were added $\left(\beta_{4}-\beta_{7}\right)$ to describe an additional cohort of orders. Cohort, a binary indicator variable, held a value of either 0 or 1 when the model was used to describe the treatment or comparison group, respectively. The coefficients $\beta_{4}-\beta_{7}$ described the treatment group, and $\beta_{0}-\beta_{3}$ described the comparison group. $\beta_{4}$ was the difference in the number of baseline orders per 10,000 patient days between treatment and comparison groups; $B_{5}$ represented the difference between the estimated ordering trends of treatment and comparison groups; and $B_{6}$ indicated the difference in immediate changes in the number of orders per $10,000 \mathrm{pa}$ tient days in the 2 groups following the intervention.

The number of orders per week was recorded for each medicine, which enabled a large number of data points to be included in our analyses. This allowed for more accurate and stable estimates to be made in our regression model. A total of 143 data points were collected for each study group, 116 before and 27 following each intervention.

All analyses were conducted by using STATA version 13.1 (StataCorp LP, College Station, TX).

\section{RESULTS}

Initial results pertaining to $9 \mathrm{IV}$ medications were examined (Table). Following the implementation of cost messaging, no significant changes were observed in order frequency or trend for IV formulations of eculizumab, calcitonin, levetiracetam, linezolid, mycophenolate, ribavirin, voriconazole, and levothyroxine (Figures 1 and 2). However, a significant decrease in the number of oral ribavirin orders (Figure 2), the control group for the IV form, was observed $(-16.3$ orders per 10,000 patient days; $P=.004 ; 95 \% \mathrm{CI},-27.2$ to $-5.31)$

From March 26, 2015, to January 21, 2016, the use of IV pantoprazole was restricted at our facility due to a national shortage, requiring clinical pharmacy review and approval. Additionally, on September 15, 2014, oncology order sets that allowed antibiotic prescribing without an infectious disease provider's approval replaced oral voriconazole with oral posaconazole. Consequently, both IV pantoprazole and oral voriconazole exhibited a sharp, sustained decrease in ordering following these policy changes (Figure 3). Although an apparent difference in ordering followed the policy changes, no significant change was noted in IV pantoprazole and oral voriconazole following our intervention.

\section{DISCUSSION}

Our results suggest that the passive strategy of displaying cost alone was not effective in altering prescriber ordering 


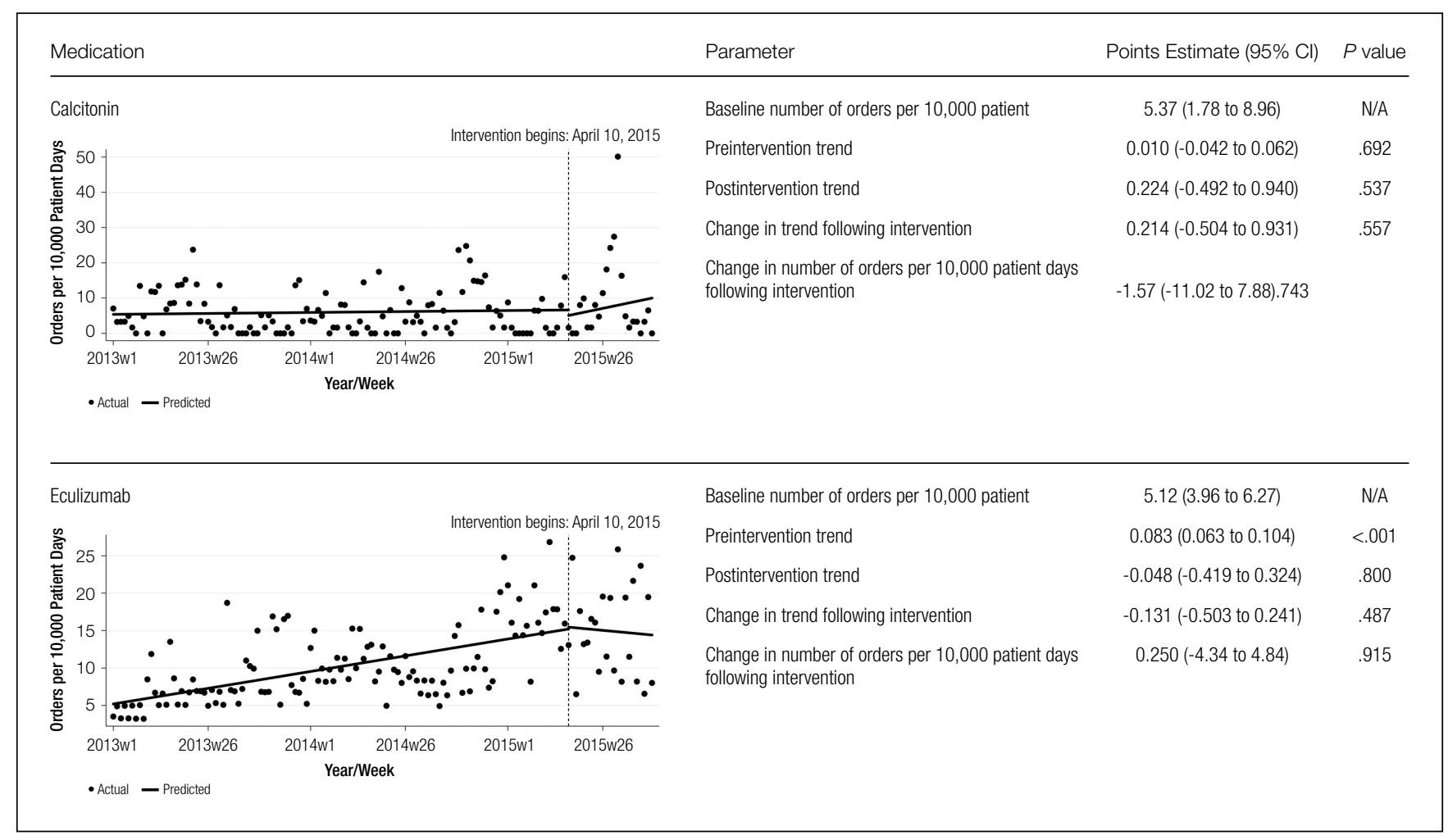

FIG 1. Ordering time trends for medications with no alternatives (parameter estimates from 2-group segmented regression analyses). Analyses in this section are divided by a vertical, dashed line. This line represents the time period at which cost messaging was implemented.

Note: N/A, not applicable

patterns for the selected medications. This may be due to a lack of awareness regarding direct financial impact on the patient, importance of costs in medical decision-making, or a perceived lack of alternatives or suitability of recommended alternatives. These results may prove valuable to hospital and pharmacy leadership as they develop strategies to curb medication expense.

Changes observed in IV pantoprazole ordering are instructive. Due to a national shortage, the IV form of this medication underwent a restriction, which required approval by the pharmacy prior to dispensing. This restriction was instituted independently of our study and led to a $73 \%$ decrease from usage rates prior to policy implementation (Figure 3). Ordering was restricted according to defined criteria for IV use. The restriction did not apply to oral pantoprazole, and no significant change in ordering of the oral formulation was noted during the evaluated period (Figure 3).

Oral voriconazole also exhibited a marked reduction in ordering of $46 \%$ following a change in hospital formulary that occurred independent of our study. The change in formulary involved a replacement of oral voriconazole with oral posaconazole in several oncology order sets, allowing antifungal administration without authorization by an infectious disease clinician. A dramatic reduction in ordering resulted from the removal of oral voriconazole from these order sets. Figure 3 represents the effect of the policy change on ordering. The frequency of orders for IV voriconazole, which was the target of our study intervention, did not change during this period (Figure 3 ).

The dramatic effect of policy changes, as observed with pantoprazole and voriconazole, suggests that a more active strategy may have a greater impact on prescriber behavior when it comes to medication ordering in the inpatient setting. It also highlights several potential sources of confounding that may introduce bias to cost-transparency studies.

This study has multiple limitations. First, as with all observational study designs, causation cannot be drawn with certainty from our results. While we were able to compare medications to their preintervention baselines, the data could have been impacted by longitudinal or seasonal trends in medication ordering, which may have been impacted by seasonal variability in disease prevalence, changes in resistance patterns, and annual cycling of house staff in an academic medical center. While there appear to be potential seasonal patterns regarding prescribing patterns for some of the medications included in this analysis, we also believe the linear regressions capture the overall trends in prescribing adequately. Nonstationarity, or trends in the mean and variance of the outcome that are not related to the intervention, may introduce bias in the interpretation of our findings. However, we believe the parameters included in our models, namely the immediate change in the intercept following the intervention and the change in the trend of the rate of prescribing over time from pre-to postintervention, provide 


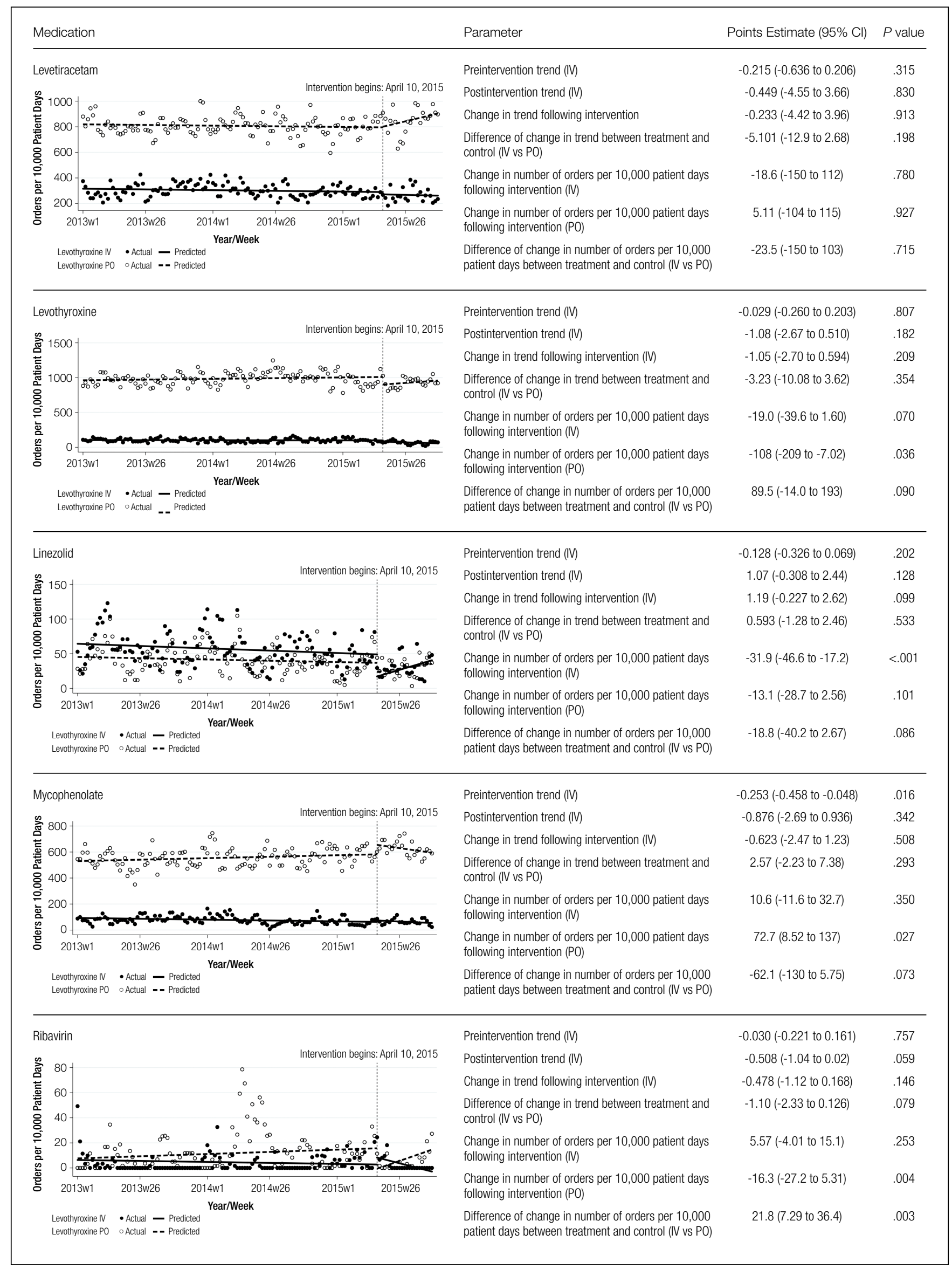

FIG 2. Ordering time trends for medications with alternatives (parameter estimates from 2-group segmented regression analyses). Analyses in this section are divided by a vertical, dashed line. This line represents the time period at which cost messaging was implemented. 


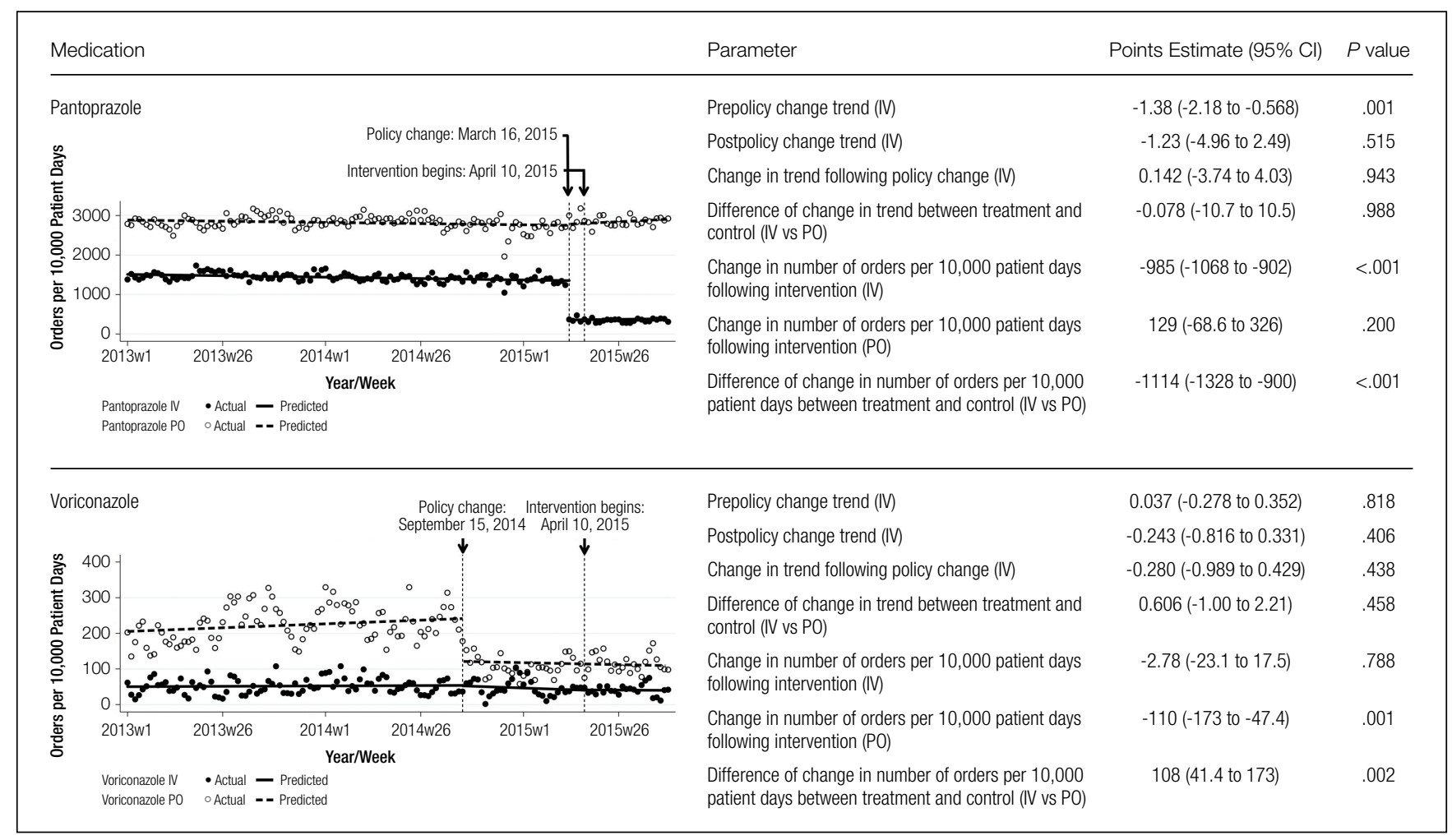

FIG 3. Ordering time trends for medications that underwent policy change (parameter estimates from 2-group segmented regression analyses). Graphical depictions of the analyses in this section are divided by a vertical, dashed line. The latter line represents the time period of our intervention, and the former line represents the time period at which the institutional policy change (relevant to that medication) took place.

Note: IV, intravenous, NA, not applicable; PO, oral

substantial protections from faulty interpretation. Our models are limited to the extent that these parameters do not account for nonstationarity. Additionally, we did not collect data on dosing frequency or duration of treatment, which would have been dependent on factors that are not readily quantified, such as indication, clinical rationale, or patient response. Thus, we were not able to evaluate the impact of the intervention on these factors.

Although intended to enhance internal validity, comparison groups were also subject to external influence. For example, we observed a significant, short-lived rise in oral ribavirin (a control medication) ordering during the preintervention baseline period that appeared to be independent of our intervention and may speak to the unaccounted-for longitudinal variability detailed above.

Finally, the clinical indication and setting may be important. Previous studies performed at the same hospital with price displays showed a reduction in laboratory ordering but no change in imaging. ${ }^{18,19}$ One might speculate that ordering fewer laboratory tests is viewed by providers as eliminating waste rather than choosing a less expensive option to accomplish the same diagnostic task at hand. Therapeutics may be more similar to radiology tests, because patients presumably need the treatment and often do not have the option of simply not ordering without a concerted effort to re- evaluate the treatment plan. Additionally, in a tertiary care teaching center such as ours, a junior clinician, oftentimes at the behest of a more senior colleague, enters most orders. In an environment in which the ordering prescriber has more autonomy or when the order is driven by a junior practitioner rather than an attending (such as daily laboratories), results may be different. Additionally, institutions that incentivize prescribers directly to practice cost-conscious care may experience different results from similar interventions.

We conclude that, in the case of medication cost messaging, a strategy of displaying cost information alone was insufficient to affect prescriber ordering behavior. Coupling cost transparency with educational interventions and active stewardship to impact clinical practice is worthy of further study.

Disclosures: The authors state that there were no external sponsors for this work. The Johns Hopkins Hospital and University "funded" this work by paying the salaries of the authors. The author team maintained independence and made all decisions regarding the study design, data collection, data analysis, interpretation of results, writing of the research report, and decision to submit it for publication. Dr. Shermock had full access to all the study data and takes responsibility for the integrity of the data and accuracy of the data analysis.

\section{References}

1. Berwick DM, Hackbarth AD. Eliminating Waste in US Health Care. JAMA. 2012;307(14):1513-1516. 
2. PricewaterhouseCoopers' Health Research Institute. The Price of Excess: Identifying Waste in Healthcare Spending. http://www.pwc.com/us/en/healthcare/publications/the-price-of-excess.html. Accessed June 17, 2015.

3. Allan GM, Lexchin J, Wiebe N. Physician awareness of drug cost: a systematic review. PLoS Med. 2007;4(9):e283.

4. Feldman LS, Shihab HM, Thiemann D, et al. Impact of providing fee data on laboratory test ordering: a controlled clinical trial. JAMA Intern Med. 2013;173(10):903-908.

5. Durand DJ, Feldman LS, Lewin JS, Brotman DJ. Provider cost transparency alone has no impact on inpatient imaging utilization. J Am Coll Radiol. 2013;10(2): 108-113.

6. Silvestri MT, Bongiovanni TR, Glover JG, Gross CP. Impact of price display on provider ordering: A systematic review. J Hosp Med. 2016;11(1):65-76.

7. Ornstein SM, MacFarlane LL, Jenkins RG, Pan Q, Wager KA. Medication cost information in a computer-based patient record system. Impact on prescribing in a family medicine clinical practice. Arch Fam Med. 1999;8(2):118-121.

8. Guterman JJ, Chernof BA, Mares B, Gross-Schulman SG, Gan PG, Thomas D. Modifying provider behavior: A low-tech approach to pharmaceutical ordering. $J$ Gen Intern Med. 2002;17(10):792-796.

9. McNitt JD, Bode ET, Nelson RE. Long-term pharmaceutical cost reduction using a data management system. Anesth Analg. 1998;87(4):837-842.

10. Horrow JC, Rosenberg H. Price stickers do not alter drug usage. Can J Anaesth. 1994:41(11):1047-1052.
11. Guterman JJ, Chernof BA, Mares B, Gross-Schulman SG, Gan PG, Thomas D. Modifying provider behavior: A low-tech approach to pharmaceutical ordering. J Gen Intern Med. 2002;17(10):792-796.

12. McNitt JD, Bode ET, Nelson RE. Long-term pharmaceutical cost reduction using a data management system. Anesth Analg. 1998;87(4):837-842.

13. Ornstein SM, MacFarlane LL, Jenkins RG, Pan Q, Wager KA. Medication cost information in a computer-based patient record system. Impact on prescribing in a family medicine clinical practice. Arch Fam Med. 1999;8(2):118-121.

14. Horrow JC, Rosenberg H. Price stickers do not alter drug usage. Can J Anaesth. 1994;41(11):1047-1052.

15. Jandoc R, Burden AM, Mamdani M, Levesque LE, Cadarette SM. Interrupted time series analysis in drug utilization research is increasing: Systematic review and recommendations. J Clin Epidemiol. 2015;68(8):950-56.

16. Linden A. Conducting interrupted time-series analysis for single- and multiple-group comparisons. Stata J. 2015;15(2):480-500.

17. Linden A, Adams JL. Applying a propensity score-based weighting model to interrupted time series data: improving causal inference in programme evaluation. J Eval Clin Pract. 2011;17(6):1231-1238.

18. Feldman LS, Shihab HM, Thiemann D, et al. Impact of providing fee data on laboratory test ordering: a controlled clinical trial. JAMA Intern Med. 2013;173(10):903-908.

19. Durand DJ, Feldman LS, Lewin JS, Brotman DJ. Provider cost transparency alone has no impact on inpatient imaging utilization. J Am Coll Radiol. 2013;10(2):108-113. 